\title{
Decodificação via Cancelamento Sucessivo de Interferência Generalizado para Sistemas de Acesso Múltiplo Não Ortogonal
}

\author{
Rafael Campello, Gabriel Carlini, Carlos E. C. Souza, Cecilio Pimentel, Daniel P. B. Chaves
}

\begin{abstract}
Resumo-Neste trabalho é apresentado um decodificador com regiões de decisão adaptáveis para sistemas de acesso múltiplo não ortogonal uplink com três usuários que faz uso do pressuposto de conhecimento dos ganhos do canal. São apresentados cenários em que o desempenho do decodificador proposto supera o método de cancelamento sucessivo de interferência tradicional e é capaz de replicar resultados obtidos por métodos de aprendizagem profunda propostos recentemente na literatura.
\end{abstract}

Palavras-Chave-Acesso múltiplo não ortogonal, cancelamento sucessivo de interferência, taxa de erro de símbolo, regiões de decisão.

Abstract-In this work, we propose a decoder with adaptable decision regions for the three-user uplink non-orthogonal multiple access (NOMA) scheme by exploiting the knowledge of the channel gains. We present scenarios in which the proposed decoder outperforms the traditional successive interference cancellation method and is able to replicate the performance of deep learning based methods recently proposed in the literature.

Keywords-Non-orthogonal multiple access (NOMA), successive interference cancellation (SIC), symbol error-rate, decision regions.

\section{INTRODUÇÃO}

Técnicas baseadas em compartilhamento dos recursos de canal têm ganhado destaque em decorrência da necessidade de garantir maior eficiência espectral nas próximas gerações de sistemas de comunicações sem fio [1]. Dentre estas, a técnica de acesso múltiplo não ortogonal (NOMA, non-orthogonal multiple access) no domínio da potência é uma importante componente para este fim [2], [3]. Com esta técnica, os sinais de cada usuário com diferentes níveis de potência são superpostos e o cancelamento sucessivo de interferência (SIC, successive interference cancellation) é um método de decodificação usualmente empregado.

Em sistemas NOMA, o fenômeno de patamar de erro é reportado na literatura [4]-[6], em que a alocação inadequada de potência entre os usuários pode potencializar a propagação de erros na decodificação SIC [7]. Em especial, os resultados de [4] sugerem que ao utilizar símbolos piloto e considerar diferenças pequenas para os parâmetros de ganhos do canal, um modelo baseado em rede neural é capaz de superar decisivamente a decodificação SIC tradicional.

Os autores são do Departamento de Eletrônica e Sistemas, Universidade Federal de Pernambuco, Recife-PE, e-mails: \{rafael.campello, gabriel.cmsilva2, carlos.ecsouza, cecilio.pimentel, daniel.chaves\}@ufpe.br. O presente trabalho foi realizado com o apoio do $\mathrm{CNPq}$, Conselho Nacional de Desenvolvimento Científico e Tecnológico.
Neste trabalho, consideramos o cenário em que três usuários com modulação QPSK se comunicam com uma estação base, chamado de uplink NOMA. Propomos uma modificação na região de decisão referente à primeira etapa do algoritmo SIC que é capaz de justificar e replicar a melhoria de desempenho reportada para algoritmos baseados em aprendizagem profunda [4] para a situação considerada em que o decodificador conhece os ganhos do canal. O método proposto permite que técnicas clássicas possam ser utilizadas na análise de desempenho e implementação do decodificador, contribuindo para a interpretabilidade dos resultados e potenciais extensões para cenários generalizados. $\mathrm{O}$ cálculo analítico de desempenho de decodificadores SIC para diferentes cenários de NOMA pode ser visto em [5], [8]-[14]. Também é apresentada uma expressão analítica da taxa de erro de símbolo (SER, symbol error rate) para a etapa em que a região de decisão modificada é utilizada.

O restante deste trabalho é estruturado da seguinte forma. Definimos o sistema de comunicação e apresentamos o decodificador proposto na Seção II. Na Seção III, uma análise analítica para a SER é apresentada. Na Seção IV apresentamos os resultados sobre o comportamento da SER para os cenários considerados. Por fim, na Seção V discutimos as considerações finais deste trabalho.

\section{O SISTEMA DE COMUNICAÇÃO}

Consideramos um sistema uplink NOMA consistindo de uma estação base e três usuários. O sinal recebido $\boldsymbol{y}=$ $\left(y_{I}, y_{Q}\right)$ é dado por

$$
\boldsymbol{y}=\sum_{i=1}^{3} h_{i}^{\prime} \sqrt{P_{i}} \boldsymbol{x}_{i}+\boldsymbol{n}
$$

em que $h_{i}^{\prime}$ é o ganho do canal entre o usuário $i$ e a estação base, $P_{i}$ é a potência transmitida pelo usuário $i, \boldsymbol{x}_{i}$ é o símbolo QPSK de energia unitária transmitido pelo usuário $i, \boldsymbol{x}_{i} \in \mathcal{A}=\{( \pm B, \pm B)\}$ e $B=\sqrt{2} / 2, \boldsymbol{n}=\left(n_{I}, n_{Q}\right)$ é o ruído aditivo Gaussiano branco (AWGN, additive white Gaussian noise) modelado como uma variável aleatória Gaussiana de média zero e variância $N_{0} / 2$ por dimensão. Definimos $h_{i} \triangleq h_{i}^{\prime} \sqrt{P_{i}}$ como o termo multiplicativo do símbolo $\boldsymbol{x}_{i}$ e consideramos que os $h_{i}$ são conhecidos pelo decodificador e fixos durante a transmissão. Estes são nomeados tal que $h_{1} \geq h_{2} \geq h_{3}$.

Tradicionalmente, o sinal recebido pela estação base é decodificado utilizando a técnica SIC, em que os sinais são 
decodificados seguindo a ordenação dos $h_{i}$ [4]. Os símbolos estimados, $\hat{\boldsymbol{x}}_{i}, i \in\{1,2,3\}$, são obtidos iterativamente de acordo com

$$
\hat{\boldsymbol{x}}_{i}=\underset{j}{\arg \min }\left|\boldsymbol{y}_{i}-h_{i} \boldsymbol{x}_{j}\right|^{2}
$$

em que $\boldsymbol{x}_{j} \in \mathcal{A}$ e $\boldsymbol{y}_{i}=\boldsymbol{y}_{i-1}-h_{i-1} \hat{\boldsymbol{x}}_{i-1}$, com inicialização $\boldsymbol{y}_{1} \triangleq \boldsymbol{y}$.

\section{A. Decodificador SIC Proposto}

Consideramos os 64 possíveis sinais $\boldsymbol{y}$ na ausência do ruído AWGN em (1) para diferentes configurações dos parâmetros $h_{i}$. No caso de três usuários, a desigualdade $\mathcal{D}: h_{1}>h_{2}+h_{3}$ é determinante para a distribuição desses possíveis sinais no plano I/Q. Se $\mathcal{D}$ for satisfeita, todos os símbolos transmitidos pelo usuário 1 pertencem aos respectivos quadrantes para quaisquer símbolos transmitidos pelos usuários 2 e 3 . Caso contrário, indicado por $\overline{\mathcal{D}}$, a interferência dos usuários 2 e 3 pode ser suficiente para modificar o quadrante do símbolo transmitido pelo usuário 1 .

A Fig. 1 apresenta as constelações para os casos $\mathcal{D}$ (Fig. 1a) e $\overline{\mathcal{D}}$ (Fig. 1b). Em ambas constelações a coloração dos símbolos indica o símbolo transmitido pelo usuário 1 . A coloração do fundo indica as regiões de decisão utilizadas para ambos os casos $\mathcal{D}$ e $\overline{\mathcal{D}}$ (usando como critério a distância euclidiana mínima). A Fig. 1b justifica intuitivamente a presença do patamar de erro para a situação em que a desigualdade $\mathcal{D}$ não é satisfeita e o SIC tradicional é utilizado na decodificação. Para o cenário mostrado, 28 dos 64 símbolos transmitidos pelo usuário 1 são mapeados para fora do respectivo quadrante, resultando em erro de decodificação na primeira iteração do algoritmo SIC, e consequentemente, causando propagação de erro na decodificação dos usuários 2 e 3 .

A primeira etapa do decodificador proposto é, portanto, a avaliação de $\mathcal{D}$. Caso a desigualdade seja satisfeita o SIC tradicional é mantido para o usuário 1 . Para o caso $\overline{\mathcal{D}}$, a estimativa do símbolo transmitido para este usuário $\hat{\boldsymbol{x}}_{1}$ utiliza regiões de decisão modificadas, seguindo a situação da Fig. 1b. As demais iterações são efetuadas conforme o algoritmo SIC, visto que a determinação correta do símbolo do primeiro usuário é suficiente para retirar o patamar de erro da decodificação dos usuários 2 e 3 , já que $h_{2}>h_{3}$. Não é considerada a situação em que $h_{1}=h_{2}+h_{3}$, visto que para esta condição existem combinações de símbolos transmitidos que são mapeados para o mesmo ponto.

No cenário da Fig. 1b é preciso realizar a determinação do limiar que separa as regiões de decisão com diferentes colorações. Como um exemplo, consideramos dois símbolos indicados com círculos vermelhos na Fig. 1b. A componente em fase destes é $B h_{1}-B h_{2}+B h_{3}$ e $-B h_{1}+B h_{2}+B h_{3}$, o que indica que o decodificador deve utilizar o limiar $L=$ $B h_{3}$ que separa estas regiões. Pela simetria da constelação, o mesmo limiar pode ser encontrado para separar outras regiões de decisão, além do limiar trivial zero que separa os quatro quadrantes.

O procedimento SIC-Modificado é apresentado no Algoritmo 1. A sua entrada é o conjunto de três $h_{i}$ e $\boldsymbol{y}=$ $\left(y_{I}, y_{Q}\right)$ e sua saída são os símbolos decodificados $\hat{\boldsymbol{x}}_{1}, \hat{\boldsymbol{x}}_{2}, \hat{\boldsymbol{x}}_{3}$.

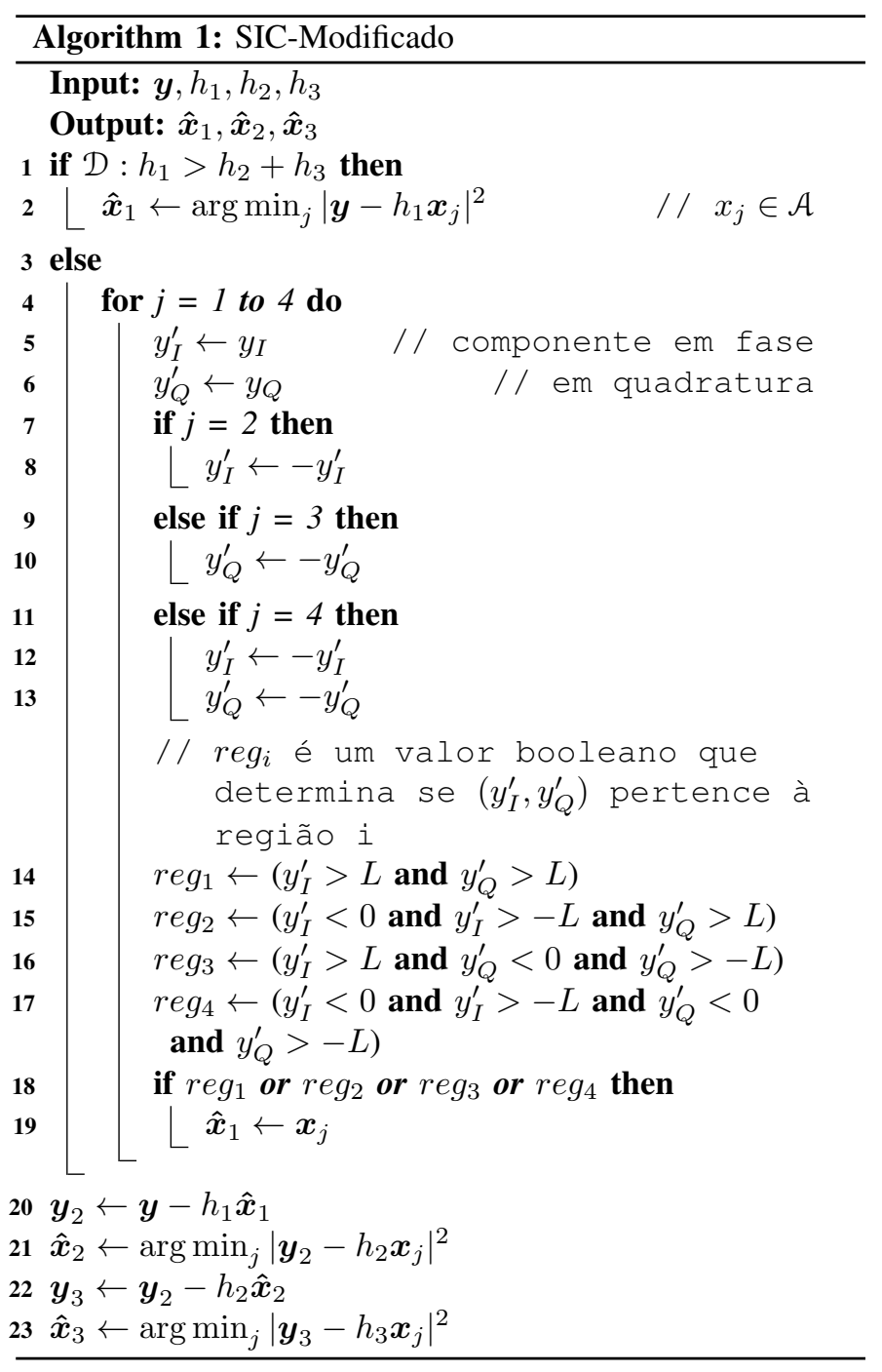

No pseudocódigo fornecido, a linha número 1 avalia a desigualdade $\mathcal{D}$ e decide qual região de decisão utilizar para o usuário 1 . O loop na linha número 4 busca determinar $\hat{\boldsymbol{x}}_{1}$ ao avaliar em qual das 16 regiões disjuntas da Fig. $1 b \boldsymbol{y}$ pertence. Para tanto o If da linha 7 realiza reflexões das componentes $\mathrm{I} / \mathrm{Q}$, o que possibilita que as avaliações de pertencimento às regiões reg1, reg2, reg3, reg4 nas linhas 14, 15, 16 e 17 possam ser realizadas. O restante do procedimento, a partir da linha 20 , é idêntico ao SIC.

\section{ANÁLISE}

Nesta seção, apresentamos uma expressão para a SER do algoritmo SIC-Modificado para a decodificação do usuário 1 em função dos parâmetros $h_{i}$ e da relação sinal ruído (SNR, signal to noise ratio). O desempenho referente a este usuário influencia diretamente no desempenho dos demais usuários devido à propagação de erro no SIC, como é visto na Seção IV. Dessa forma, a expressão analítica fornecida para o desempenho do Algoritmo 1 serve como referência para o desempenho geral do sistema proposto.

É necessário considerar diferentes expressões condicionadas à veracidade de $\mathcal{D}$. Como ambas derivações são análogas, 


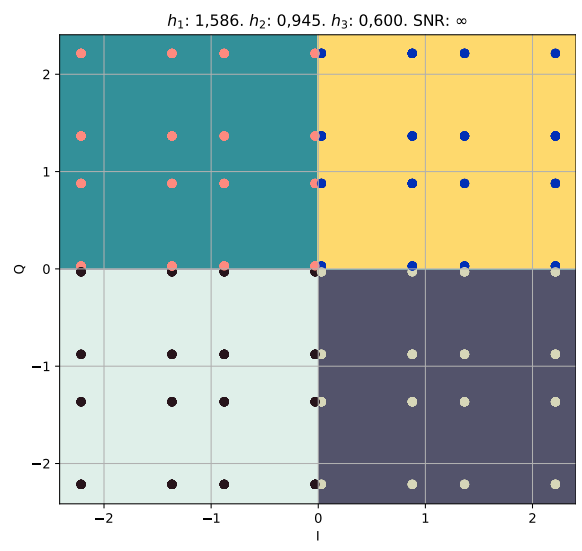

(a) $\mathcal{D}: h_{1}>h_{2}+h_{3}$

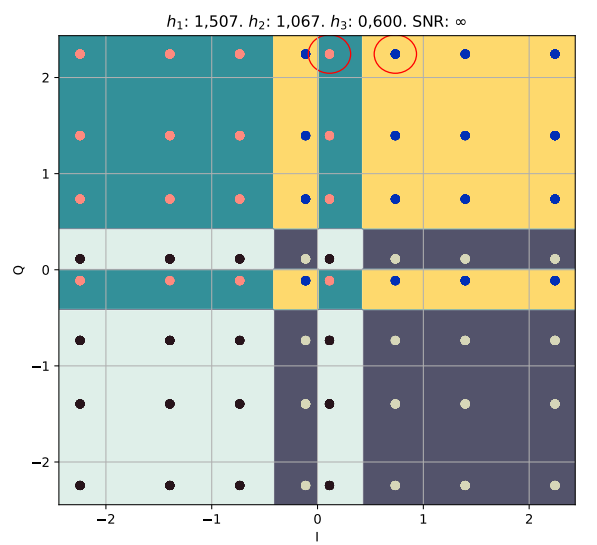

(b) $\overline{\mathcal{D}}: h_{1}<h_{2}+h_{3}$

Fig. 1. Constelação recebida para todas as 64 combinações possíveis de símbolos transmitidos para uma dada realização de $h_{1}, h_{2}, h_{3}$ para os casos $\mathcal{D}$ (Fig. 1a) e $\overline{\mathcal{D}}$ (Fig. 1b). A coloração do fundo indica as regiões de decisão.

apresentamos o cálculo para o caso $\overline{\mathcal{D}}$, situação da Fig. 1b, em que as regiões de decisão modificadas são empregadas. Como a expressão é obtida para valores de $h_{i}$ fixos, calculamos a probabilidade de uma decisão correta para o usuário 1 ao fixar o símbolo transmitido, $P\left(c_{1} \mid \overline{\mathcal{D}}, \boldsymbol{x}_{1}=(B, B)\right)$. Então consideramos a simetria do problema ao dividir os 16 símbolos resultantes em 10 casos, como indicado na Fig. 2. Assumimos que os símbolos possuem igual probabilidade de serem transmitidos. Por simetria, $P\left(c_{1} \mid \overline{\mathcal{D}}\right)=P\left(c_{1} \mid \overline{\mathcal{D}}, \boldsymbol{x}_{1}=\right.$ $(B, B))$. Então

$$
\begin{aligned}
& P\left(c_{1} \mid \overline{\mathcal{D}}\right)= \\
& =\frac{1}{16} \sum_{\boldsymbol{x}_{a} \in \mathcal{A}} \sum_{\boldsymbol{x}_{b} \in \mathcal{A}} P\left(c_{1} \mid \overline{\mathcal{D}}, \boldsymbol{x}_{1}=(B, B), \boldsymbol{x}_{2}=\boldsymbol{x}_{a}, \boldsymbol{x}_{3}=\boldsymbol{x}_{b}\right) \\
& =\frac{1}{16}\left\{P\left(c_{1} \mid \# \mathbf{1}\right)+P\left(c_{1} \mid \# \mathbf{5}\right)+P\left(c_{1} \mid \# \mathbf{7}\right)+P\left(c_{1} \mid \# \mathbf{1 0}\right)\right. \\
& +2\left[P\left(c_{1} \mid \# \mathbf{2}\right)+P\left(c_{1} \mid \# \mathbf{3}\right)+P\left(c_{1} \mid \# \mathbf{4}\right)+P\left(c_{1} \mid \# \mathbf{6}\right)\right. \\
& \left.\left.+P\left(c_{1} \mid \# \mathbf{8}\right)+P\left(c_{1} \mid \# \mathbf{9}\right)\right]\right\} .
\end{aligned}
$$

Expressões para cada probabilidade indicada em (3) em função de $h_{1}, h_{2}, h_{3}$ e SNR $\triangleq \rho \triangleq 1 / N_{0}$ são mostradas no apêndice. A SER condicional para o usuário 1 é $P\left(e_{1} \mid \overline{\mathcal{D}}\right)=1-P\left(c_{1} \mid\right.$ $\overline{\mathcal{D}})$. Para cada um dos dez casos, desprezamos a probabilidade do ruído AWGN levar um símbolo para outra região de mesma coloração. Esta consideração é razoável para situações práticas de SNR, conforme é visto na Seção IV, em que verificamos que a expressão encontrada concorda com os resultados de simulação. $\mathrm{O}$ procedimento análogo também pode ser feito para encontrar $P\left(e_{1} \mid \mathcal{D}\right)$, resultando numa expressão para a SER válida para toda entrada do Algoritmo 1.

\section{RESUltados}

Nesta seção, os resultados são apresentados para $h_{3}=1$ e variações nos parâmetros $h_{1}, h_{2}$ e SNR. A modificação de $h_{3}$ resulta em resultados análogos aos apresentados. Utilizamos a convenção de definir o parâmetro $h_{i}$ por meio da expressão $\left|h_{i}\right|^{2}=\left|h_{i+1}\right|^{2}+\xi_{i}(\mathrm{~dB}), i=1,2$, com $\left|h_{3}\right|^{2}=0(\mathrm{~dB})$, apresentando os resultados por meio dos parâmetros $\xi_{1}$ e $\xi_{2}$. As análises são feitas considerando resultados de simulação e

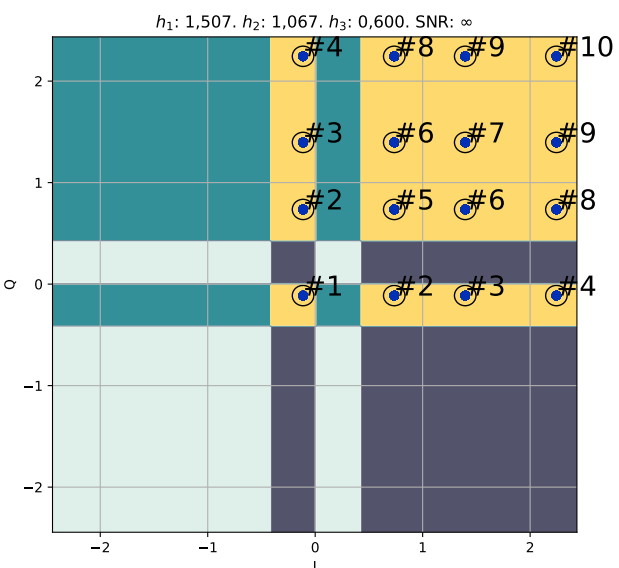

Fig. 2. Constelação resultante para $\boldsymbol{x}_{1}=(B, B)$. São indicados os dez casos considerados na derivação da expressão analítica da SER.

obtidos a partir da expressão analítica da SER do decodificador proposto para o usuário 1.

Inicialmente, é feita a comparação de desempenho do decodificador SIC tradicional, SIC-Modificado, e um decodificador baseado em rede neural (NN) análogo ao apresentado em [4], para os casos $\mathcal{D}$ e $\overline{\mathcal{D}}$. A Fig. 3 mostra a SER versus SNR para ambos os casos. Para o caso em que a desigualdade $\mathcal{D}$ é satisfeita, indicado na Fig. 3a para $\xi_{1}=\xi_{2}=5 \mathrm{~dB}$, os três decodificadores possuem desempenhos idênticos (para a faixa de SER de interesse). A Fig. 3b mostra que o desempenho do decodificador SIC-Modificado supera decisivamente o do SIC tradicional no cenário em que a desigualdade $\mathcal{D}$ não é satisfeita, para $\xi_{1}=\xi_{2}=3 \mathrm{~dB}$, devido ao uso de regiões de decisão projetadas para o caso em questão. O modelo NN possui o mesmo desempenho do SIC-Modificado, o que sugere que o ganho de desempenho da NN em relação ao SIC tradicional reportado em [4] se dá devido ao aprendizado de regiões de decisão conforme a Fig. 1b.

A Fig. 4 apresenta um mapa de calor para a SER do usuário 1 para o decodificador SIC-Modificado, em que os eixos da figura correspondem aos espaçamentos $\xi_{1}, \xi_{2}$, com a SNR fixada em $18 \mathrm{~dB}$. É indicado na figura (em linha tracejada) 


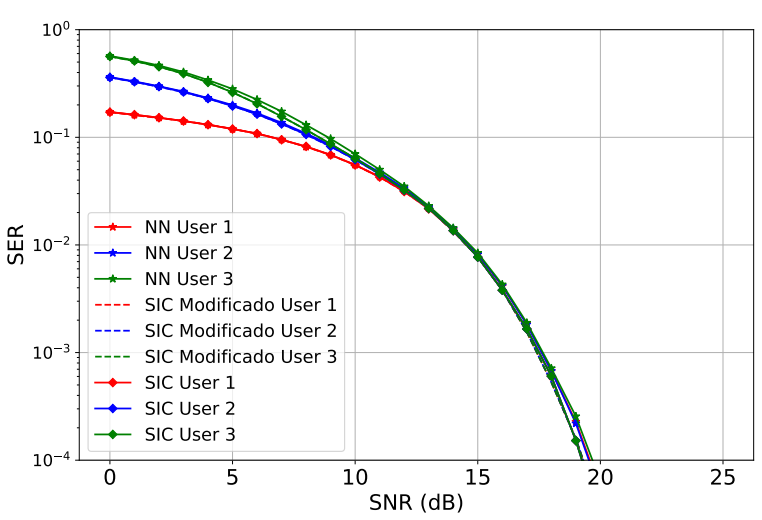

(a) $\mathcal{D}: h_{1}=3,162 . h_{2}=1,778 . h_{3}=1,000$

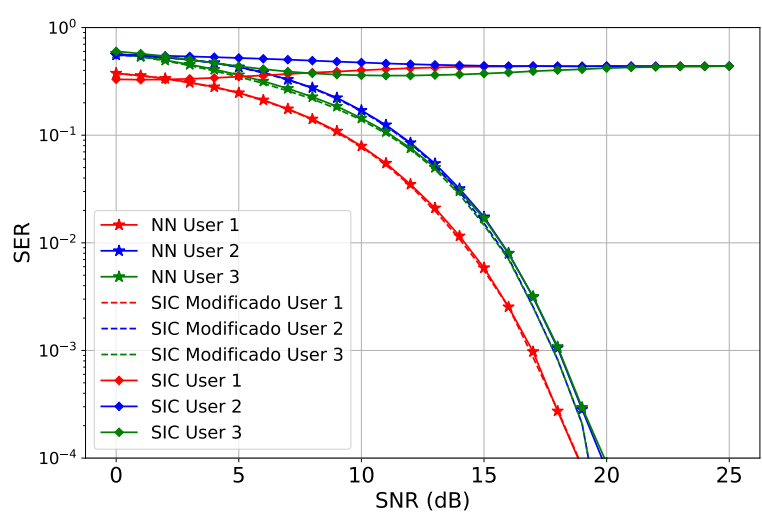

(b) $\overline{\mathcal{D}}: h_{1}=1,995 \cdot h_{2}=1,413 \cdot h_{3}=1,000$

Fig. 3. Comparação de SER referente aos decodificadores SIC tradicional, SIC-Modificado e NN. A Fig. 3a mostra o cenário em que $\xi_{1}=\xi_{2}=5 \mathrm{~dB}$ (D é satisfeita). A Fig. $3 \mathrm{~b}$ mostra o cenário em que $\xi_{1}=\xi_{2}=3 \mathrm{~dB}$ (D não é satisfeita).

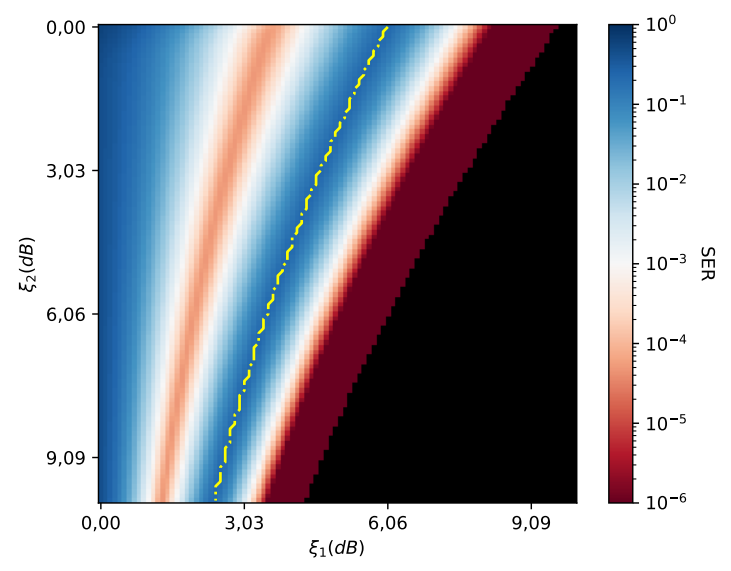

Fig. 4. Mapa de calor para a SER do usuário 1 para o decodificador SIC-Modificado. A linha tracejada indica o limiar referente à desigualdade $\mathcal{D}$. SNR $=18 \mathrm{~dB}$.

o limiar correspondente à desigualdade $\mathcal{D}$. A figura indica que o desempenho do decodificador é pior para configurações de $h_{i}$ tais que $h_{1} \approx h_{2}+h_{3}$, além disso, a desigualdade $\mathcal{D}$ divide o mapa em duas regiões, sendo possível obter melhores resultados ao se afastar do limiar indicado.

O comportamento não estritamente decrescente da SER com o aumento de $\xi_{i}$ pode ser analisado em uma dimensão com a restrição adicional $\xi \triangleq \xi_{1}=\xi_{2}$, como mostrado na Fig. 5, para ambos os casos $\mathcal{D}$ e $\overline{\mathcal{D}}$. Para o caso $\mathcal{D}$, o comportamento do SIC tradicional é observado, em que a SER diminui com o aumento do espaçamento. Já para o caso $\overline{\mathcal{D}}$ a dependência da SER com a escolha de $\xi$ não é monotônica, em que o valor ótimo do espaçamento pode ser determinado diretamente da expressão analítica para o primeiro usuário. Também podese observar que na situação $\overline{\mathcal{D}}$, o ótimo relativo ao primeiro usuário não se traduz em SER ótimo para os usuários 2 e 3.

O resultado teórico para a SER do usuário 1 possui excelente concordância com os resultados de simulação. São indicados três limiares de interesse sobre a variável $\xi$ na Fig. 5: o limiar relacionado à desigualdade $\mathcal{D}$, correspondente

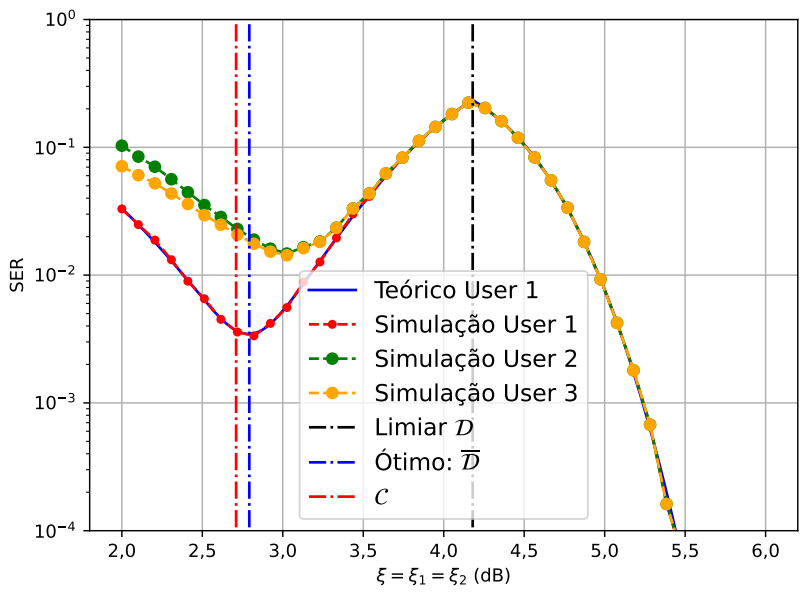

Fig. 5. SER para o decodificador S IC-Modi fi cado com a restrição $\xi=\xi_{1}=\xi_{2}$ e SNR $=15 \mathrm{~dB}$.

a $\xi=20 \log _{10}\left(\frac{1+\sqrt{5}}{2}\right) \approx 4,18 \mathrm{~dB}$, e outros dois referentes à otimalidade da escolha de $\xi$. A reta tracejada "Ótimo $\overline{\mathcal{D}}$ " indica o desempenho ótimo para a SER do usuário 1 obtido da análise analítica para o caso em que $h_{1}<h_{2}+h_{3}$, correspondente a $\xi \approx 2,79 \mathrm{~dB}$, valor em acordo com a simulação. O limiar indicado por $\mathcal{C}$ refere-se ao valor de $\xi$ correspondente ao posicionamento do ponto indicado por \#1 da Fig. 2 na coordenada $\left(\frac{-L}{2}, \frac{-L}{2}\right)$, o centro do quadrado ao qual o símbolo \#1 pertence, correspondendo a $\xi=20 \log _{10}\left(\frac{1+\sqrt{3}}{2}\right) \approx 2,71 \mathrm{~dB}$. Em outras palavras, ao tentar posicionar o símbolo indicado por \#1 da Fig. 2 no centro do quadrado correspondente, obtémse um desempenho subótimo.

\section{CONCLUSÃO}

Neste trabalho foi proposto um decodificador para o cenário de uplink NOMA com três usuários baseado em regiões de decisão adaptáveis aos parâmetros de ganho de canal. Em especial, foram analisados cenários de SERs condicionados aos casos $\mathcal{D}$ e $\overline{\mathcal{D}}$. O método representa uma maneira alternativa de se obter ganhos de desempenho previamente encontrados na literatura por meio de técnicas de aprendizagem profunda [4]. 


$$
\begin{aligned}
P\left(c_{1} \mid \# \mathbf{1}\right) & =P\left(-B h_{3}<B\left(h_{1}-h_{2}-h_{3}\right)+n_{I}<0\right) P\left(-B h_{3}<B\left(h_{1}-h_{2}-h_{3}\right)+n_{Q}<0\right) \\
& =\left(Q\left(-B \sqrt{2 \rho}\left(h_{1}-h_{2}\right)\right)-Q\left(-B \sqrt{2 \rho}\left(h_{1}-h_{2}-h_{3}\right)\right)\right)^{2} \\
P\left(c_{1} \mid \# \mathbf{2}\right) & =P\left(B h_{3}<B\left(h_{1}-h_{2}+h_{3}\right)+n_{I}<\infty\right) P\left(-B h_{3}<B\left(h_{1}-h_{2}-h_{3}\right)+n_{Q}<0\right) \\
& =\left(Q\left(-B \sqrt{2 \rho}\left(h_{1}-h_{2}\right)\right)\right)\left(Q\left(-B \sqrt{2 \rho}\left(h_{1}-h_{2}\right)\right)-Q\left(-B \sqrt{2 \rho}\left(h_{1}-h_{2}-h_{3}\right)\right)\right) \\
P\left(c_{1} \mid \# \mathbf{3}\right) & =P\left(B h_{3}<B\left(h_{1}+h_{2}-h_{3}\right)+n_{I}<\infty\right) P\left(-B h_{3}<B\left(h_{1}-h_{2}-h_{3}\right)+n_{Q}<0\right) \\
& =\left(Q\left(B \sqrt{2 \rho}\left(2 h_{3}-h_{1}-h_{2}\right)\right)\right)\left(Q\left(-B \sqrt{2 \rho}\left(h_{1}-h_{2}\right)\right)-Q\left(-B \sqrt{2 \rho}\left(h_{1}-h_{2}-h_{3}\right)\right)\right) \\
P\left(c_{1} \mid \# \mathbf{4}\right) & =P\left(B h_{3}<B\left(h_{1}+h_{2}+h_{3}\right)+n_{I}<\infty\right) P\left(-B h_{3}<B \cdot\left(h_{1}-h_{2}-h_{3}\right)+n_{Q}<0\right) \\
& =\left(Q\left(-B \sqrt{2 \rho}\left(h_{1}+h_{2}\right)\right)\right)\left(Q\left(-B \sqrt{2 \rho}\left(h_{1}-h_{2}\right)\right)-Q\left(-B \sqrt{2 \rho}\left(h_{1}-h_{2}-h_{3}\right)\right)\right) \\
P\left(c_{1} \mid \# \mathbf{5}\right) & =P\left(B h_{3}<B\left(h_{1}-h_{2}+h_{3}\right)+n_{I}<\infty\right) P\left(B h_{3}<B\left(h_{1}-h_{2}+h_{3}\right)+n_{Q}<\infty\right) \\
& =\left(Q\left(-B \sqrt{2 \rho}\left(h_{1}-h_{2}\right)\right)\right) \\
P\left(c_{1} \mid \# \mathbf{6}\right) & =P\left(B h_{3}<B\left(h_{1}+h_{2}-h_{3}\right)+n_{I}<\infty\right) P\left(B h_{3}<B\left(h_{1}-h_{2}+h_{3}\right)+n_{Q}<\infty\right) \\
& =\left(Q\left(B \sqrt{2 \rho}\left(2 h_{3}-h_{1}-h_{2}\right)\right)\right)\left(Q\left(-B \sqrt{2 \rho}\left(h_{1}-h_{2}\right)\right)\right) \\
P\left(c_{1} \mid \# \mathbf{7}\right) & =P\left(B h_{3}<B\left(h_{1}+h_{2}-h_{3}\right)+n_{I}<\infty\right) P\left(B h_{3}<B\left(h_{1}+h_{2}-h_{3}\right)+n_{Q}<\infty\right) \\
& =\left(Q\left(B \sqrt{2 \rho}\left(2 h_{3}-h_{1}-h_{2}\right)\right)\right)^{2} \\
P\left(c_{1} \mid \# \mathbf{8}\right) & =P\left(B h_{3}<B\left(h_{1}+h_{2}+h_{3}\right)+n_{I}<\infty\right) P\left(B h_{3}<B\left(h_{1}-h_{2}+h_{3}\right)+n_{Q}<\infty\right) \\
& =\left(Q\left(-B \sqrt{2 \rho}\left(h_{1}+h_{2}\right)\right)\right)\left(Q\left(-B \sqrt{2 \rho}\left(h_{1}-h_{2}\right)\right)\right) \\
P\left(c_{1} \mid \# \mathbf{9}\right) & =P\left(B h_{3}^{<B}\left(h_{1}+h_{2}+h_{3}\right)+n_{I}<\infty\right) P\left(B h_{3}<B\left(h_{1}+h_{2}-h_{3}\right)+n_{Q}<\infty\right) \\
& =\left(Q\left(-B \sqrt{2 \rho}\left(h_{1}+h_{2}\right)\right)\right)\left(Q\left(B \sqrt{2 \rho}\left(2 h_{3}-h_{1}-h_{2}\right)\right)\right) \\
P\left(c_{1} \mid \# \mathbf{1 0}\right) & =P\left(B h_{3}<B\left(h_{1}+h_{2}+h_{3}\right)+n_{I}<\infty\right) P\left(B h_{3}<B\left(h_{1}+h_{2}+h_{3}\right)+n_{Q}<\infty\right) \\
& =\left(Q\left(-B \sqrt{2 \rho}\left(h_{1}+h_{2}\right)\right)\right)^{2}
\end{aligned}
$$

Possíveis extensões do trabalho podem incluir a generalização para um número maior de usuários e possíveis regiões de decisão adaptáveis para cada usuário.

\section{APÊNDICE}

Expressões para as probabilidades $P\left(c_{1} \mid \# \boldsymbol{i}\right), i=1 \ldots 10$ são mostradas em (4)-(13). Em cada caso, é utilizada a função $Q(z)=\frac{1}{\sqrt{2 \pi}} \int_{z}^{\infty} e^{-t^{2} / 2} d t$

\section{REFERÊNCIAS}

[1] W. Saad, M. Bennis, and M. Chen, "A vision of $6 \mathrm{G}$ wireless systems: applications, trends, technologies, and open research problems," IEEE Network, vol. 34, no. 3, pp. 134-142, May 2020.

[2] Z. Ding, Y. Liu, J. Choi, Q. Sun, M. Elkashlan, I. Chih-Lin, and H. V. Poor, "Application of non-orthogonal multiple access in LTE and 5G networks," IEEE Communications Magazine, vol. 55, no. 2, pp. 185191, Feb. 2017.

[3] Y. Wang, J. Wang, D. W. Kwan Ng, R. Schober, and X. Gao, "A minimum error probability NOMA design," IEEE Transactions on Wireless Communications, vol. 20, no. 7, pp. 4221-4237, Jul. 2021.

[4] A. Emir, F. Kara, H. Kaya, and H. Yanikomeroglu, "DeepMuD: Multiuser detection for uplink grant-free NOMA IoT networks via deep learning," IEEE Wireless Communications Letters, vol. 10, no. 5, pp. 1133-1137, May 2021.

[5] M. Aldababsa, C. Göztepe, G. K. Kurt, and O. Kucur, "Bit error rate for NOMA network," IEEE Communications Letters, vol. 24, no. 6, pp. 1188-1191, Jun. 2020.

[6] F. Kara and H. Kaya, "Error probability analysis of NOMA-based diamond relaying network," IEEE Transactions on Vehicular Technology, vol. 69, no. 2, pp. 2280-2285, Feb. 2020.

[7] Q. Luo, P. Gao, Z. Liu, L. Xiao, Z. Mheich, P. Xiao, and A. Maaref, "An error rate comparison of power domain non-orthogonal multiple access and sparse code multiple access," IEEE Open Journal of the Communications Society, vol. 2, pp. 500-511, 2021.
[8] I. Lee and J. Kim, "Average symbol error rate analysis for nonorthogonal multiple access with m-ary QAM signals in Rayleigh fading channels," IEEE Communications Letters, vol. 23, no. 8, pp. 1328-1331, Aug. 2019.

[9] F. Wei, T. Zhou, T. Xu, and H. Hu, "BER analysis for uplink NOMA in asymmetric channels," IEEE Communications Letters, vol. 24, no. 11, pp. 2435-2439, Nov. 2020.

[10] F. Kara and H. Kaya, "BER performances of downlink and uplink NOMA in the presence of SIC errors over fading channels," IET Communications, vol. 12, no. 15, pp. 1834-1844, 2018.

[11] X. Wang, F. Labeau, and L. Mei, "Closed-form BER expressions of QPSK constellation for uplink non-orthogonal multiple access," IEEE Communications Letters, vol. 21, no. 10, pp. 2242-2245, Oct. 2017.

[12] Q. He, Y. Hu, and A. Schmeink, "Closed-form symbol error rate expressions for non-orthogonal multiple access systems," IEEE Transactions on Vehicular Technology, vol. 68, no. 7, pp. 6775-6789, Jul. 2019.

[13] F. Kara and H. Kaya, "Error probability analysis of non-orthogonal multiple access with channel estimation errors," in 2020 IEEE International Black Sea Conference on Communications and Networking (BlackSeaCom), May 2020, pp. 1-5.

[14] T. Assaf, A. J. Al-Dweik, M. S. E. Moursi, H. Zeineldin, and M. Al-Jarrah, "Exact bit error-rate analysis of two-user NOMA using QAM with arbitrary modulation orders," IEEE Communications Letters, vol. 24, no. 12, pp. 2705-2709, Dec. 2020. 